\title{
Rolling Bearing Fault Detection in the Initial Stage of Degradation Based upon Optimized NLM and TKEO
}

\author{
Hongru Li* \\ Mechanical Engineering College, Shijiazhuang 050003, P.R. China.
}

\author{
Baiyan Chen \\ Mechanical Engineering College, Shijiazhuang 050003, P.R. China. \\ 63850 troops of the PLA, Baicheng 137001, P.R. China.
}

\author{
He Yu \\ Mechanical Engineering College, Shijiazhuang 050003, P.R. China. \\ Yaolong Li and Hongqiang Gu \\ Mechanical Engineering College, Shijiazhuang 050003, P.R. China.
}

(Received 17 January 2017; accepted 22 September 2017)

\begin{abstract}
During the operation of rolling bearings, vibration signals contain abundant state information, which exhibits strong nonstationarity and nonlinearity. It is always arduous to detect the initial damage point during the lifetime. Non-local means (NLM) algorithm can suppress noise and highlight the components of the fault impact, but the problem lies in the determination of parameters which directly affect the result. In this paper, we proposed a signal processing method combined NLM optimized by Fruit fly Optimization Algorithm (FOA) and Teager Kaiser energy operator (TKEO) to detect the initial stage degradation of bearings. First of all, the proposed optimal NLM algorithm is used to denoise the bearing vibration signals which are gathered in the initial stage of bearing degradation. Then, the TKEO algorithm is applied to suppress the non-impulsive components and the periodic impulsive characteristics of the denoised signals are enhanced simultaneously. Furthermore, the analysis of the frequency components in the Teager energy spectrum is conducted to detect whether the bearings are abnormal or not. Experimental and comparative analyses are presented to validate the proposed method in the end.
\end{abstract}

\section{INTRODUCTION}

Rolling bearing is one of the most widely used rotating components at present. It plays an important role as "mechanical joints" in electromechanical equipment and weapon equipment. Unfortunately, rolling bearing is usually vulnerable due to the disadvantageous operation environment, so it is important to find out the hidden damage in it. ${ }^{1}$ Condition Based Maintenance (CBM) is a kind of popular maintenance method in recent years. This method can detect abnormalities and figure out whether there is tendency to malfunction according to the real-time state monitoring data so as to take further maintenance and preventive measures. ${ }^{2}$ If the damage of the rolling bearing can be detected as soon as possible, there will be enough time to work out reasonable maintenance program to further ensure the safe and reliable operation of the equipment.

The vibration signal of the rolling bearing contains abundant state information, which shows strong non-stationary and non-linear characteristics. And it is influenced by the complex vibration transmission path, serious noise disturbance, the

${ }^{*}$ Corresponding author. coupling of multi-vibrational source excitation and response and other factors. ${ }^{3}$ Especially in the early stage of bearing degradation, the shock signal caused by structural damage is difficult to detect. ${ }^{4}$ Fast Kurtogram (FK) method processes signals through a pass-band filter, which is simple and convenient to be applied in vibration signal denoising. 5 However, it should be realized that FK has a limited range of possible centre frequencies particularly when the bandwidth is wide. ${ }^{6}$ Meanwhile, there is a limitation to the accuracy improvement of extracting transient characteristics from noisy signals and recognizing machinery fault with the kurtogram method. ${ }^{7}$ As a new denoising method, non-local means (NLM) algorithm has the advantages of simple calculation, no iteration and positive performance. Therefore, it has gradually become a hotspot research topic in image processing ${ }^{8}$ and biomedical. ${ }^{9}$ However, this algorithm is still in its infancy in the processing of onedimensional vibration signals. Combining NLM with Empirical Mode Decomposition (EMD), Mien et al. ${ }^{10}$ firstly applied NLM in the field of vibration signals and the bearing fault diagnosis was realized. $\mathrm{Lv}$ et al. ${ }^{11}$ diagnosed rolling bearing fault successfully by fast NLM algorithm with the envelope spectrum analysis. Zhu et al. ${ }^{12}$ combined NLM and Local Mean 
Decomposition (LMD) to achieve the fault feature extraction of rolling beaings, which is better than just using LMD. Nevertheless, the NLM parameters are usually determined by human experience. The determination of the parameters directly affects signal denoising, which further influences the results of fault detection in the early stage of bearing degradation.

The local search performance of genetic algorithm (GA) is not satisfactory. In the late stage of parameter optimization, the convergence speed of the algorithm is relatively slow when it is close to the global optimal value and that increases the time of obtaining the global optimal parameter. ${ }^{13}$ Fruit fly Optimization Algorithm (FOA) is a kind of globaloptimization swarm intelligent algorithm proposed by PAN in $2011 .{ }^{14}$ This algorithm has the advantages of few parameters, fast calculation speed, strong ability of global optimization and easy implementation, and it has been popularized and applied in a multitude of fields. ${ }^{15,16}$ In this paper, for the purpose of maximizing the kurtosis of denoising signals, FOA is applied to select the parameters of NLM to achieve the optimal smoothing effect of vibration signals in the process of noise reduction

Teager Kaiser energy operator (TKEO) is a nonlinear difference operator. It is suitable for signal demodulation analysis in the impact detection because of its high time resolution, fast demodulation speed, and the positive ability of enhancing signal transient characteristics. ${ }^{17}$ However, TKEO demodulation method only applies to the single-component signals for amplitude modulation and frequency modulation. During the bearing operation, there are always other components associated with rotating frequency and other strong noise components in the vibration signals. ${ }^{18}$ Therefore, TKEO has no obvious effect on the demodulation of the vibration signals with low SNR. ${ }^{19}$

In order to solve the above problems, a fault detection method based on NLM optimized by FOA and TKEO in the initial stage of rolling bearing degradation is proposed in this paper. First of all, the root mean square (RMS) index is used to find the initial stage vibration signal of the whole life data. Maximization of the kurtosis of the denoised signal is taken as the optimization target. The filter parameters and structure half width parameters of NLM are optimized through FOA. The vibration signal is denoised by optimized NLM. The impact components are further enhanced through TKEO. Finally, the bearing fault detection is finished by observing the frequency components of Teager energy spectrum, and the type of fault is further determined.

The paper is organized as follows: Section 2 presents FOA search for optimal parameters in details, NLM algorithm and the optimized NLM algorithm by FOA and GA. Section 3 presents TKEO in details. Section 4 presents a proposed method based upon optimized NLM and TKEO for rolling bearing fault detection in the initial stage of degradation. A positive discussion and analysis of the experimental results are also obtained in this section by comparing the proposed method with TKEO demodulation directly on origin data. Finally, our conclusions are provided in Section 5.

\section{OPTIMIZED NLM ALGORITHM BY FOA}

\subsection{NLM Algorithm}

NLM algorithm is a kind of image processing method based on block similarity theory proposed by A BUADES et al. ${ }^{20}$ This method is developed from the neighbourhood filtering. The core idea is to find as much as possible similarity to the weighted average in a large range called the search window. There is a large amount of redundant information in nature, which contains a number of similar components. The different modules will be repeated, and the theory was found based on the fact. The vibration signal of the rolling bearing contains abundant state information. It is bound to repeat a certain period of shock signal when the bearing is damaged in operation. The noise superimposed on the structural similarity block is random. Therefore, we can remove the noise by weighted average. NLM algorithm in one dimensional signal is as follows: ${ }^{10}$

Assuming that $x(t)$ is a real signal, $n(t)$ is the noise signal, the actual signal can be expressed as:

$$
y(t)=x(t)+n(t) .
$$

In the non local mean filtering algorithm, $X$ is used to estimate the original signal $x$, which is obtained by the weighted average of all the similar structures in the search domain. Therefore, the estimated value can be expressed as:

$$
X(s)=\frac{1}{Z(t)} \sum_{t \in N(s)} \omega(s, t) y(t)
$$

Where, $N(s)$ represents the search area with the sample point $t$ as the centre. $Z(t)$ represents the summation of all blocks of similar structure. $\omega(s, t)$ represents similarity. $y(t)$ represents actual signal containing noise. In formula (2):

$$
\begin{gathered}
Z(t)=\sum_{t \in N(s)} \omega(s, t) ; \\
\omega(s, t)=\exp \left(-\frac{\sum_{\delta \in \Delta}(y(s+\delta)-y(t+\delta))^{2}}{2 L_{\Delta} \lambda^{2}}\right) \\
\equiv \exp \left(-\frac{d^{2}(s, t)}{2 L_{\Delta} \lambda^{2}}\right) ; \\
0 \leq \omega(s, t) \leq 1 ; \\
\sum_{t} \omega(s, t)=1 ;
\end{gathered}
$$

where $\lambda$ represents filter parameter. It controls the attenuation rate of $\omega(s, t)$, and determines the smoothness of the denoised signal. $\Delta=[s-p, s+p]$ represents the target structure block taking $\mathrm{s}$ as the centre. $L_{\Delta}=[t-p, t+p]$ represents the similar structure block taking $t$ as the centre. Weight between two similar blocks is measured with $d^{2}(s, t)$ which denotes the sum of the squared point-to-point dissimilarity.

The target recovery structure block is assumed to be $A$. Three key parameters of NLM are the half width of structure 


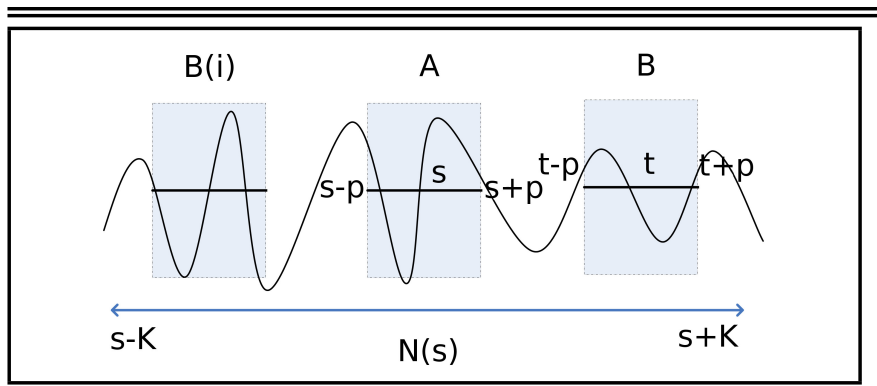

Figure 1. Illustration of NLM parameter relationship.

block $P$, the half width of search area $K$ and the filter parameter $\lambda$. The meaning of each parameter is demonstrated in Fig. 1. The target structure block A and the similar structure block B are matched by a search window, which has the same length, and the similarity is calculated by $\omega(s, t)$. The similar structure block $B$ is found in the search domain to estimate the target block $A$ centre point $s$.

\subsection{Fruit Fly Optimization Algorithm}

First, the position of the fruit fly population is initialized. According to the coordinate position of the fruit fly population, each of the flies in the initial population searches the direction and distance of food through their smell sense. The specific location of the food can not be learned. Consequently, the distance between the fruit fly and the origin need to be calculated. The reciprocal of the distance was used as the food concentration decision value of the fruit fly individual. The food concentration in each individual location in the fly population is calculated, and the maximum value of the food concentration and the position coordinates of the fruit fly are preserved according to the concentration. The fly population flies to the coordinates with vision. The coordinate of the fruit fly individual is initialized again in the new position, and the above process is repeated until the location of the food is found. The flow chart of FOA is manifested in Fig. 2.

The detailed steps of the algorithm are as follows: ${ }^{14}$

1. The fruit fly population is assumed to be consisting of $N$ fruit fly individual. Above all, the location of fruit fly population $(x, y)$ is initialized. The location of fruit fly individual is further initialized according to $(x, y)$. The individuals search food with smell sense.

$$
\left\{\begin{array}{l}
x_{i}=x+L_{k} \\
y_{i}=x+L_{k}
\end{array} ;\right.
$$

Where, $L_{k}$ is the random step value in the step interval $[-L, L] . \quad L$ is the maximum step size that the fruit fly individual can fly in search of food with smell.

2. The distance between individual $i(1, \ldots, N)$ and food is calculated. The decision value of taste concentration $D e_{i}$ is further calculated through the distance.

$$
\begin{gathered}
d_{i}=\sqrt{x_{i}^{2}+y_{i}^{2}} ; \\
D e_{i}=\frac{1}{d_{i}} ;
\end{gathered}
$$

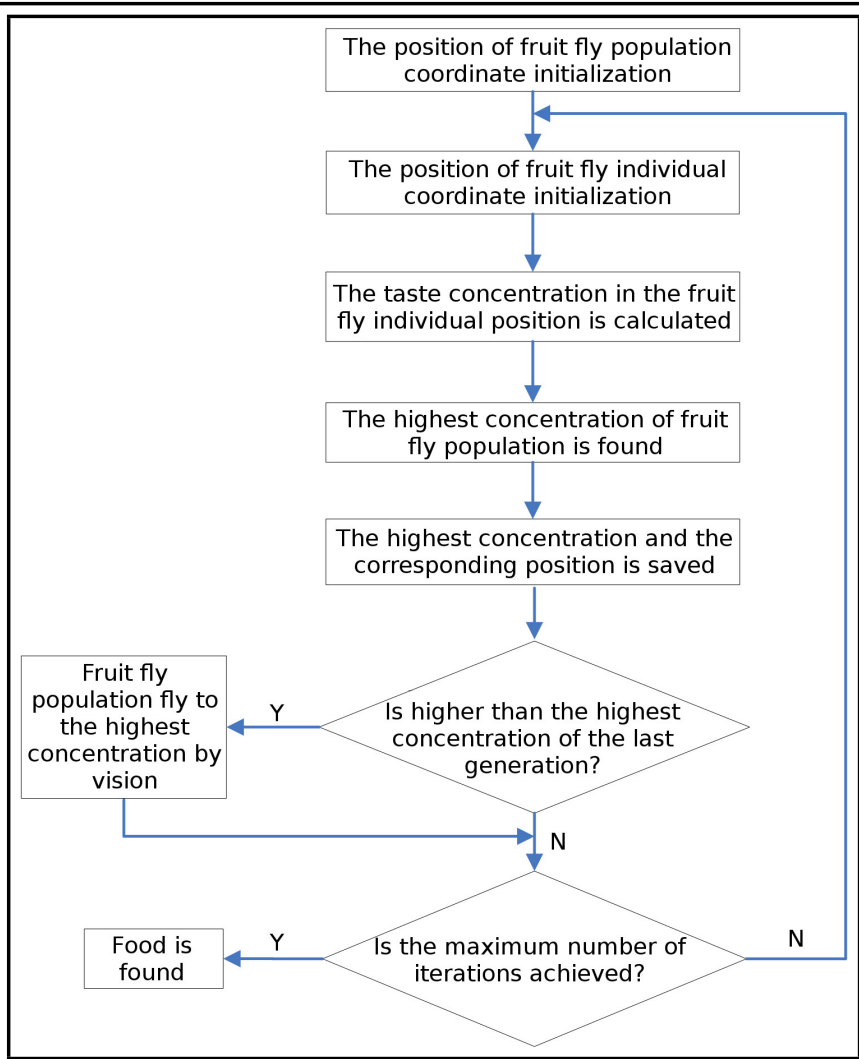

Figure 2. The flow chart of FOA implement.

3. The taste concentration obtained by individuals smell sense is calculated with smell concentration decision function based upon $D e_{i}$.

$$
\operatorname{sm}_{i}=\text { fitness function }\left(D e_{i}\right) ;
$$

4. The individual of the highest taste concentration is found.

$$
[\text { Tsm index }]=\max (s m) \text {; }
$$

where, Tsm is obtained highest concentration. index is the order number of fruit fly individual obtaining highest concentration. $s m$ is a set of fruit fly population taste concentration. Whether the taste concentration is better than the previous generation is determined. If Yes, the step (5) is executed. Otherwise, repeat steps (2) to (4).

5. The location of the best taste concentration and obtained individual is preserved, and fruit fly population fly to the position with vision.

$$
\begin{gathered}
s m T=T s m \\
\left\{\begin{array}{l}
x_{T}=x(\text { index }) \\
y_{Y}=y(\text { index })
\end{array}\right.
\end{gathered}
$$

6. Whether to meet the preset conditions is determined (the fixed fitness value according to the actual problem or the maximum number of iterations). If Yes, the best location of food is found. Otherwise, return step (2).

\subsection{Optimized NLM Algorithm by FOA}

It is crucial to determine the parameter when NLM algorithm is applied to denoised signal. A greater search area can 
obtain a better result. However, increasing the search area will cost much time. It is more formidable to determine $p$ and $\lambda$. Ville and Kocher aimed at simulation on research Sure method. ${ }^{21,22}$ It can achieve best result that $\lambda$ is determined as $0.5 \sigma . \sigma$ is noise standard deviation. Nevertheless, $\sigma$ is more formidable to be determined in the actual signal, and it can only be determined by the way of estimation. FOA is a kind of swarm intelligence algorithm for global optimization. In order to achieve the best denoising effect, the optimal $p$ and $\lambda$ can be determined by FOA. The accuracy of the initial stage of degradation fault detection is further ensured.

Kurtosis is the dimensionless parameter in time domain analysis, which describes maximum impulsiveness of a waveform. Its mathematical expression is as follows:

$$
K=\frac{E(x-\mu)^{4}}{\sigma^{4}}
$$

where, $\mu$ is the mean value of signal $x . \sigma$ is the standard deviation of signal $x$.

Kurtosis is mainly used to measure the degree that the vibration signal amplitude deviates from the normal distribution. The kurtosis is about 3 when the signal is approximate normal distribution, while the kurtosis increased obviously as the signal has more impact component. ${ }^{23}$

Based upon the above analysis, the maximum kurtosis is taken as optimized target in denoised signal, and the filter parameters and structure half width parameters of NLM are optimized through FOA. The best denoising effect is achieved and more impact components are highlighted. The optimization steps are as follows:

1. The search area of NLM is determined as following principle: If the signal length is short $(N<4000), N(S)$ is taken as the signal length to make the search area covering the whole signal range. The more weighted average similar blocks is performed to obtain, which makes good use of redundant information of the signal itself. If the signal length is long $(N>4000), N(S)$ is determined as: $1 / 2 * N \leq N(s) \leq 2 / 3 * N$, considering the problem of calculating time. ${ }^{11}$

2. The maximum kurtosis is taken as optimized target in denoised signal. The filter parameters $\lambda$ and structure half width parameters $p$ of NLM are optimized through FOA.

3. The optimized parameters are put into NLM, and then the original vibration signal denoising is completed with FOA-NLM.

\subsection{Optimized NLM Algorithm by GA}

$N(S)$ is also determined as: $1 / 2 * N \leq N(s) \leq 2 / 3 * N$, which is the same as optimized through FOA. The free parameters $p$ and $\lambda$ greatly affect the denoising effect of NLM algorithm. GA is used to search for better combination of the parameters in NLM, and it can obtain the optimal solution after a series of iterative computations. The optimization steps are as follows:
1. Initialization: Randomly generate an initial population of chromosomes which represent the values of parameters $p$ and $\lambda$.

2. Calculating the fitness function: The maximum kurtosis is taken as fitness function in denoised signal.

3. GA operators: Selection, crossover and mutation operators generate the offspring of the existing population in GA. Offspring replaces the old population and forms a new population in the next generation by the three operations, the evolutionary process proceeds until stop conditions are satisfied.

4. Suppressing noise: The optimized parameters by GA are put into NLM, and then the original vibration signal denoising is completed by GA-NLM.

\section{TEAGER KAISER ENERGY OPERATOR}

Signal energy is traditionally defined as the square of the amplitude of the signal. However, the impact component may be submerged when the impact amplitude is small. TKEO estimates the total energy required for signal source to generate a dynamic signal through the nonlinear combination of the instantaneous value of the signal and its derivative. Compared with the traditional energy definition, the product between the square of frequency and signal amplitude replace the square of the amplitude. The frequency of the transient shock is higher. Accordingly, TKEO can effectively enhance the transient shock component. TKEO has the advantage of simple calculation and it is suitable for processing AM-FM signals which has a slow change of instantaneous frequency and relative high SNR. ${ }^{24}$

For a AM-FM signal $x(t), x(t)=a(t) \cos [\phi(t)]$. The TKEO is defined as follows: ${ }^{25}$

$$
\psi[x(t)]=[\dot{x}(t)]^{2}-x(t) \ddot{x}(t)
$$

where, $\dot{x}(t)=\frac{d x(t)}{d t}, \ddot{x}(t)=\frac{d^{2} x(t)}{d t^{2}}$.

It can be demonstrated that the discrete version of the TKEO is:

$$
\psi[x(n)]=[x(n)]^{2}-x(n-1) x(n+1) .
$$

Instantaneous frequency $\omega(n)$ is the difference function of $\phi(n)$ :

$$
\omega(n)=\phi(n)-\phi(n-1) ;
$$

$x(n)=a(n) \cos [\phi(n)]$ substitute in Eq. (15):

$$
\begin{array}{r}
\psi[x(n)]=a(n)^{2} \cos ^{2} \phi(n) \\
-a(n-1) \cos [\phi(n-1)] a(n+1) \cos [\phi(n+1)] . \\
\text { Suppose } y(n)=x(n)-x(n-1) \\
\psi[y(n)]=4 a(n)^{2} \sin ^{2} \frac{\omega(n)}{2} \sin ^{2} \omega(n) .
\end{array}
$$

The instantaneous frequency and instantaneous amplitude are both calculated:

$$
\begin{aligned}
& \omega(n)=\arccos \left[1-\frac{\psi[y(n)]}{2 \psi[x(n)}\right] \\
& |a(n)|=\sqrt{\frac{\psi[x(n)}{1-\left[1-\frac{\psi[y(n)]}{2 \psi[x(n)]}\right]}} .
\end{aligned}
$$




\section{ROLLING BEARING FAULT DETECTION IN THE INITIAL STAGE OF DEGRADA- TION BASED UPON OPTIMIZED NLM AND TKEO}

\subsection{Rolling Bearing Fault Detection Method in the Initial Stage of Degradation}

The procedure of the proposed method that achieves rolling bearing fault detection in the initial stage of degradation is shown as follows:

1. Initial stage of degradation signal is determined with RMS index of the run-to-failure. Maximization of the kurtosis of the denoised signal is taken as the optimization target. The filter parameters and structure half width parameters of NLM are optimized through FOA.

2. Original vibration signal is denoised with optimized NLM.

3. The denoised signal is processed with TKEO. The transient shock components are enhanced and non impact components are suppressed.

4. Fault detection is finished through spectrum analysis for the processed signal, and the fault type is further determined based on the frequency component in the Teager energy spectrum.

\subsection{Rolling Bearing Inner Race Fault Detec- tion in the Initial Stage of Degradation}

In this paper, the bearing whole life tester provided by Hangzhou Bearing Test \& Research Center (HBRC), and the experimental platform (ABLT-1A) is manifested in Fig. 3. A load of $6.6 \mathrm{kN}$ is appended on the bearings to accelerate the bearing to failure. The type of bearing used in the experiment is 6204, which is applied to mechanical equipment widely, and its structure parameters are displayed in Table 1. The rotation speed is kept at $1500 \mathrm{rpm}$. The whole lifetime of the testbearing is $9800 \mathrm{~min}$. Inner race failure occurred at the end of the test to failure experiment. YD-1 acceleration sensors are adopted in data acquisition module. Data were collected every 10 minutes, and 20480 points are collected every time. The data sampling rate is $25.6 \mathrm{kHz}$. A total of 980 sets data are collected.

Inner race failure occurred in the experiment. Inner race fault frequency is calculated:

$$
f=\frac{z f_{r}}{2}\left(1+\frac{D_{b}}{D_{c}} \cos \alpha\right)
$$

where, $z$ represents ball number. $f_{r}$ represents rotating frequency. $D_{b}$ represents Ball diameter. $D_{c}$ represents Pitch diameter. $\alpha$ represents contact angle. The bearing structure parameters in Table 1 are entered into Eq. (21). Inner race fault frequency is obtained as $123.7 \mathrm{~Hz}$.

The RMS of whole lifetime vibration data is demonstrated in Fig. 4 to search for the degradation initial stage vibration. RMS begins to increase at $6970 \mathrm{~min}$ as we can see from the

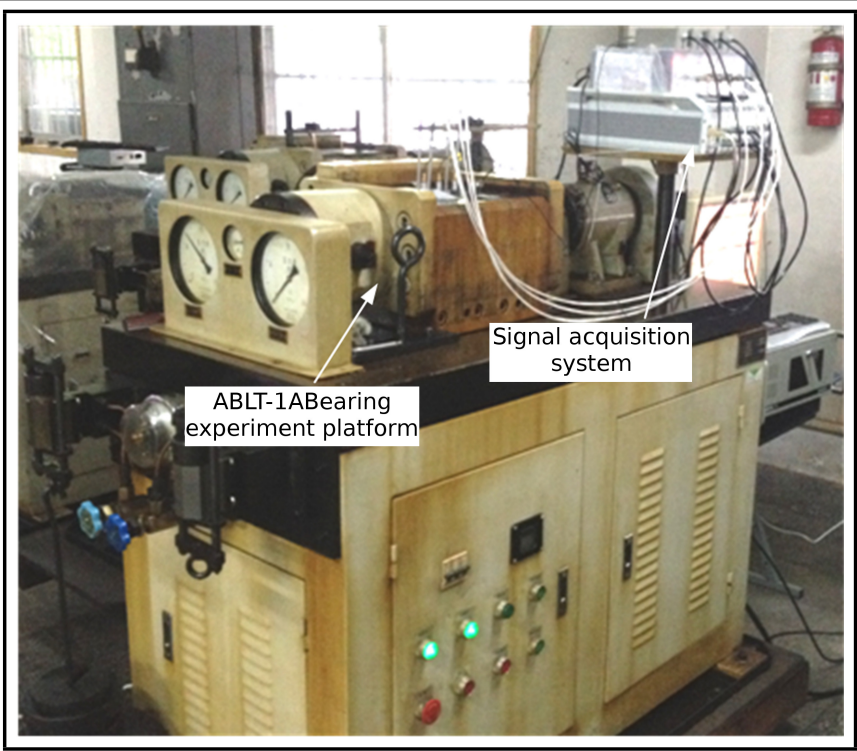

Figure 3. ABLT-1A bearing experiment platform.

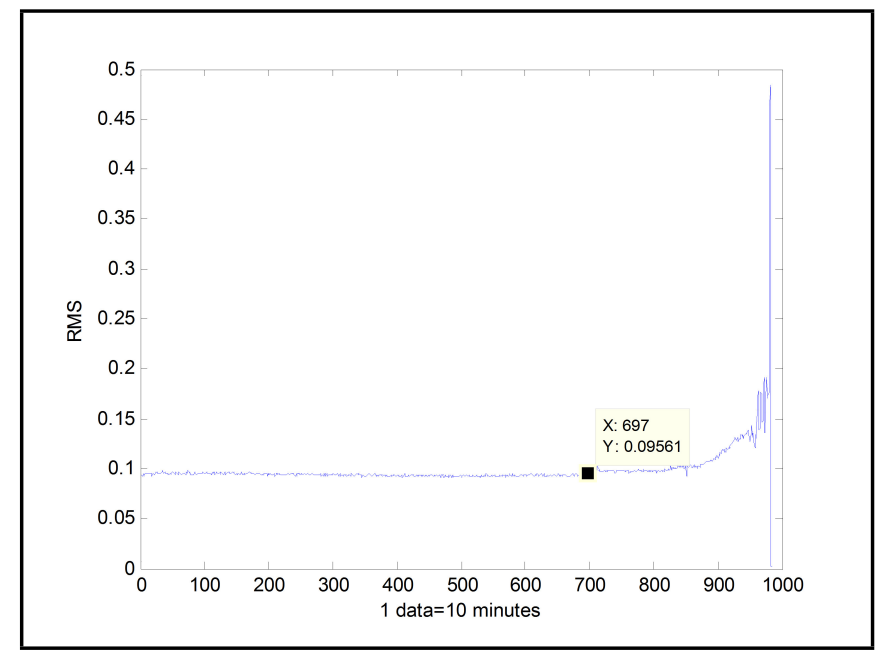

Figure 4. The RMS of whole lifetime.

Fig. 4. The data at this time is set as the vibration signal of the initial stage of degradation. Its time domain figure and squared envelope spectrum is shown as Fig. 5. Whether the fault has occurred is not determined from the squared envelope spectrum. Fault frequency is submerged in noise, and fault cannot be detected effectively.

The parameters of NLM are optimized through FOA. In order to highlight the advantages of FOA in optimizing parameters, the results of GA optimization are also listed. Parameters of the optimizing algorithms are displayed in Table 2. The fitness curve of parameter optimization is shown in Fig. 6. Optimized results are demonstrated in Table 3 (Operating platform for R2011b Matlab, the main computer is configured as: Intel(R) Core(TM) i5-4590 CPU 3.30 GHz, 4 G memory). Results indicate that FOA is far faster than the GA, and the final denoising effect is better than GA. The experimental results are in agreement with the theory that GA is slow in the late stage of parameter optimization and easy to fall into local optimal solution.

The search area of NLM $N(S)=10^{4}$ is determined. Original vibration signal at $6970 \mathrm{~min}$ is denoised with optimized 
Table 1. Structure parameters of rolling bearing.

\begin{tabular}{|c|c|c|c|c|c|c|}
\hline Type & Pitch diameter & Ball diameter & Ball number & Contact angle & Rated radial load & Mass \\
\hline 6204 & $335 \mathrm{~mm}$ & $7.938 \mathrm{~mm}$ & 8 & $0^{\circ}$ & $6.6 \mathrm{kN}$ & $0.11 \mathrm{~kg}$ \\
\hline
\end{tabular}

Table 2. Parameters of the optimizing algorithms.

\begin{tabular}{|c|c|c|c|c|}
\hline Algorithm & $\begin{array}{c}\text { The maximum number } \\
\text { of iterations }\end{array}$ & $\begin{array}{c}\text { Population } \\
\text { size }\end{array}$ & $\begin{array}{c}\text { Initial } \\
\text { coordinates }\end{array}$ & $\begin{array}{c}\text { Step } \\
\text { value }\end{array}$ \\
\hline FOA & 200 & 20 & $x=[5 \cdot \operatorname{rand}(1,1)]$ & 2 \\
\cline { 3 - 4 } & 200 & 20 & \multicolumn{2}{|c|}{ Crossover probability: 0.7} \\
\cline { 3 - 5 } GA & 20 & \multicolumn{2}{|c|}{ Mutation probability: 0.1} \\
\hline
\end{tabular}

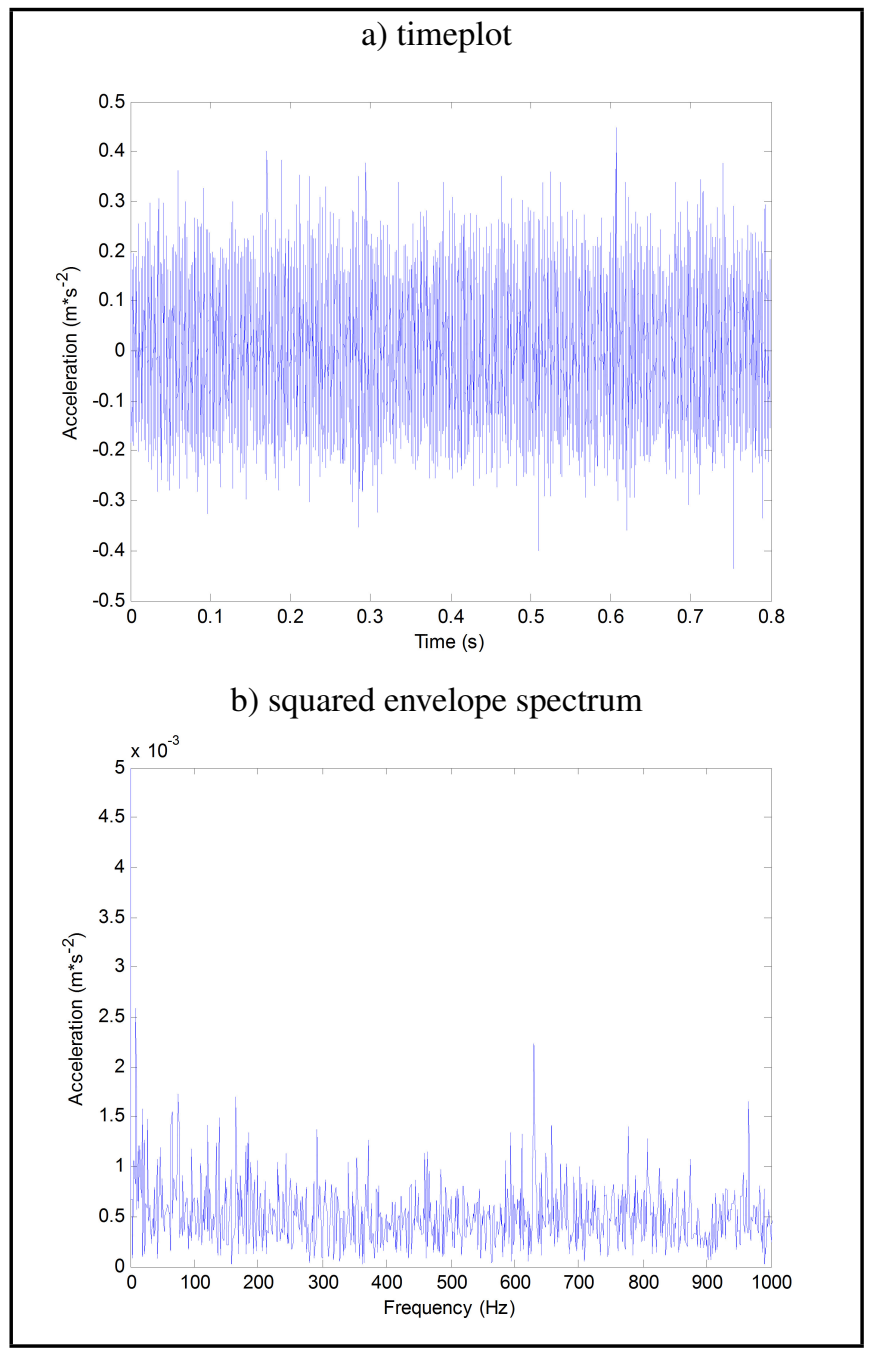

Figure 5. The time domain and squared envelope spectrum at $6970 \mathrm{~min}$.

NLM. Time domain waveform after optimized FOA-NLM is manifested in Fig. 7. The kurtosis of the denoised signal is obviously increased. The impact component has been improved accordingly. The denoised signal is processed with TKEO, and TKEO frequency spectrum after FOA-NLM is shown in Fig. 8. The fault frequency and the doubling can be detected from the figure. There are some deviations between the fault frequency and the theoretical value on trial, which is mainly caused by the instability of the speed. It can not affect the detection results, and the results demonstrate the effectiveness of the proposed method in this paper.

Time domain waveform after optimized GA-NLM is also demonstrated in Fig. 9. It is arduous to find out the more pleasurable optimization method only from the time domain

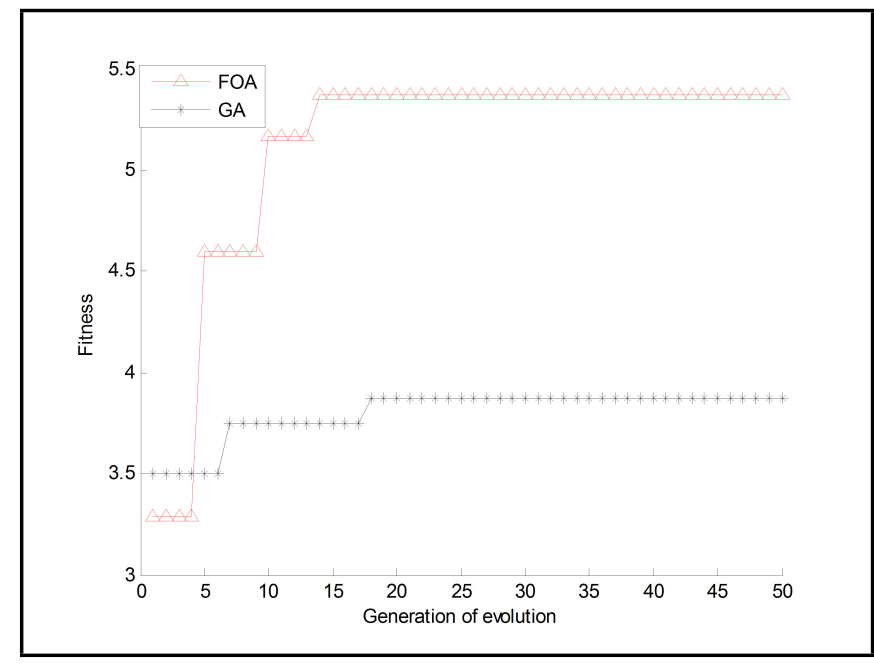

Figure 6. The fitness curve of parameter optimization.

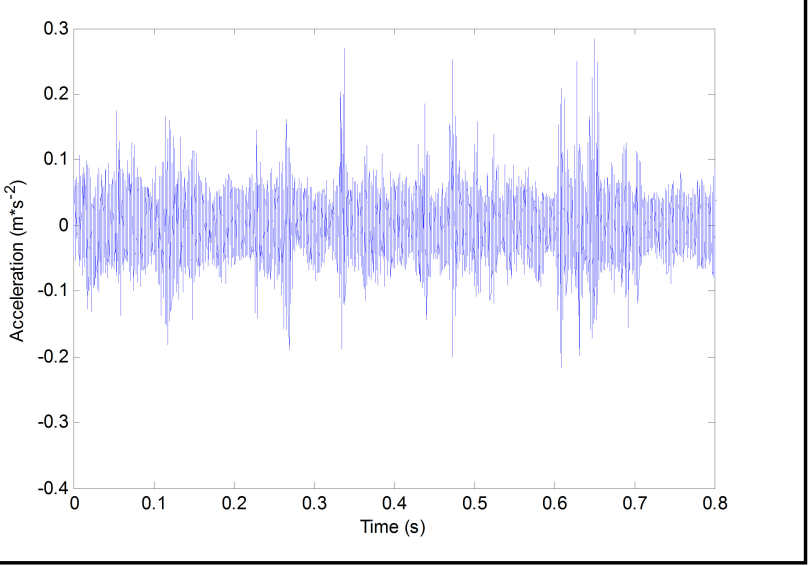

Figure 7. Timeplot after FOA-NLM.

waveform. The denoised signal is processed with TKEO, and TKEO frequency spectrum after GA-NLM is shown in Fig. 10. The fault frequency is submerged in noise. Compared with Fig. 8 and Fig. 10, the noise suppressed by GA-NLM method is obviously stronger than FOA-NLM method, for which GA falls into local optimal solution. GA-NLM can hardly discover fault information and little or nothing detect the fault. Consequently, we can determine that the final denoising effect is less impressive by GA-NLM compared with the proposed method.

In order to verify the proposed method more favourable compared with the FK method. The corresponding kurtogram is manifested in Fig. 11 and the maximum kurtosis is $K_{4}$, indicated by the black rectangle in the figure. The filter signal with 
Table 3. Comparison of FOA and GA optimization algorithms results.

\begin{tabular}{|c|c|c|c|c|}
\hline Algorithm & $\begin{array}{c}\text { Optimized results } \\
(p, \lambda)\end{array}$ & $\begin{array}{c}\text { Time in optimization } \\
\text { algorithms }(\mathrm{s})\end{array}$ & $\begin{array}{c}\text { Convergent iterative } \\
\text { number }\end{array}$ & $\begin{array}{c}\text { The kurtosis of } \\
\text { denoised signal }\end{array}$ \\
\hline FOA & $(20,0.035)$ & 2.8841 & 14 & 5.3693 \\
\hline GA & $(49,0.142)$ & 19.8294 & 26 & 3.8702 \\
\hline
\end{tabular}

Table 4. Parameters of the rolling bearing.

\begin{tabular}{|c|c|c|c|c|}
\hline Type & Pitch diameter & Ball diameter & Ball number & Contact angle \\
\hline ZA2115 & $71.5 \mathrm{~mm}$ & $8.4 \mathrm{~mm}$ & 16 & $15.17^{\circ}$ \\
\hline
\end{tabular}

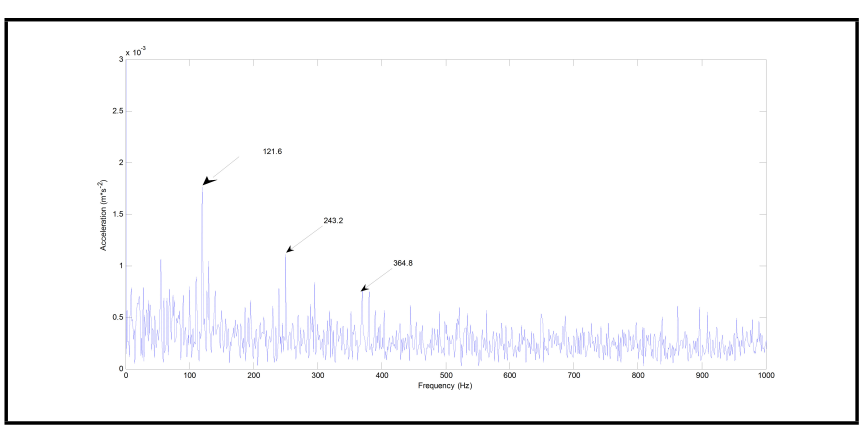

Figure 8. TKEO frequency spectrum after FOA-NLM.

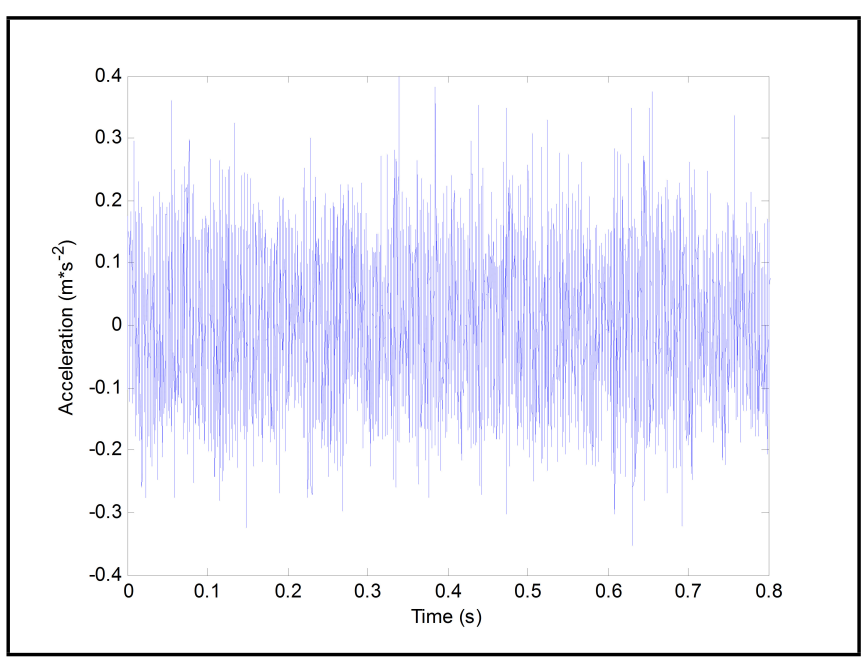

Figure 9. Timeplot after GA-NLM.

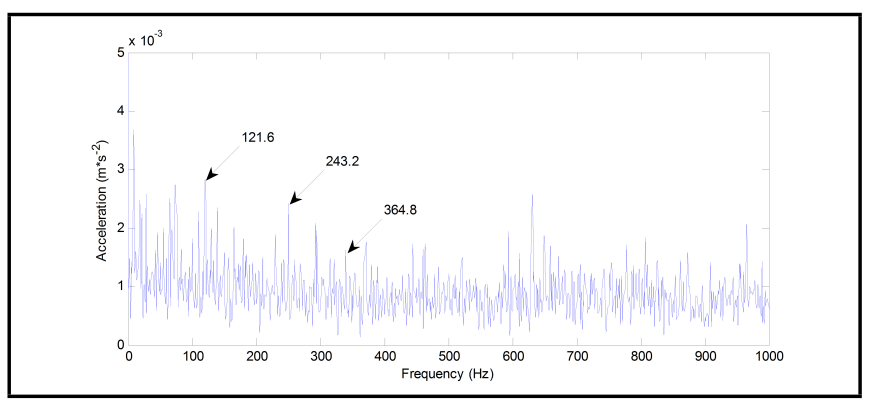

Figure 10. TKEO frequency spectrum after GA-NLM.

the maximum kurtosis displayed in Fig. 12, and it is processed by TKEO in Fig. 13. The fault frequency is not particularly obvious though it can be barely detected, and the detection effect is not as outstanding as the proposed method.

In order to explain the necessity of this method, TKEO demodulation method that is directly carried out in origin signal is taken as comparison. The spectrum of TKEO demodulation directly on the $6970 \mathrm{~min}$ data is shown in Fig. 14. In contrast to Fig. 8 and Fig. 14, we can conclude that TKEO is its sensitiv-

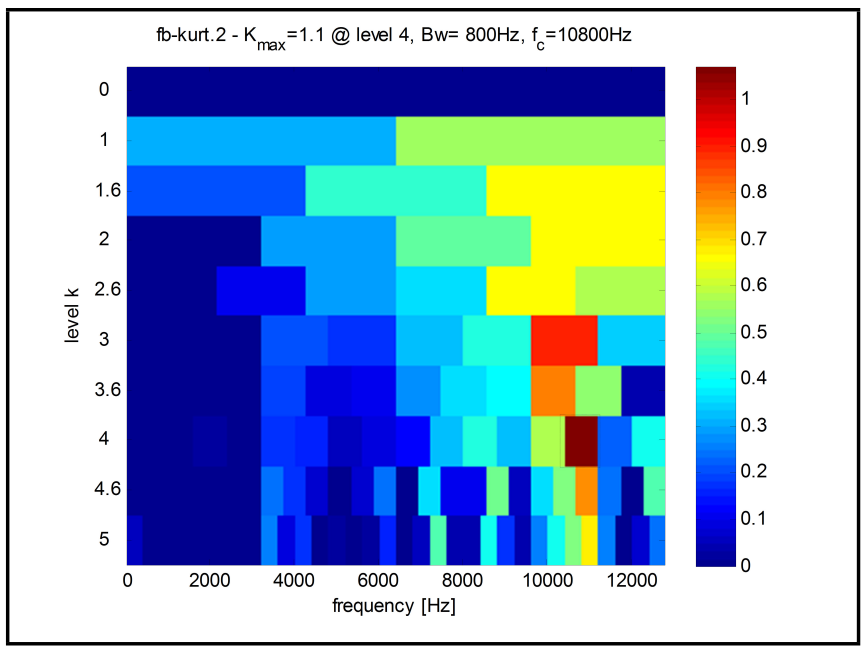

Figure 11. Kurtogram for the experimental bearing at $6970 \mathrm{~min}$.

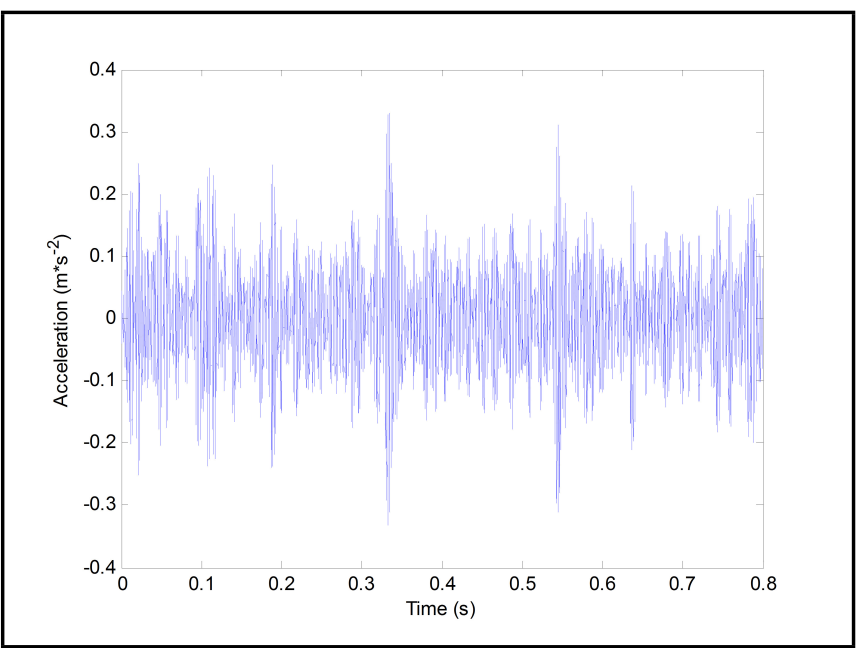

Figure 12. Filter signal timeplot.

ity to noise. In the early stage of degradation, the fault signal is extremely weak. The instantaneous frequency and amplitude are estimated through TKEO. Nevertheless, fault can not be detected as a result of fault frequency submerged in noise. This is consistent with the conclusions given in reference. ${ }^{19}$ Accordingly, the necessity of the proposed method is verified.

\subsection{Rolling Bearing Outer Race Fault Detec- tion in the Initial Stage of Degradation}

Cincinnati University Intelligent Maintenance Systems (IMS) experimental data is selected to study rolling bearing outer race fault detection in the initial stage of degradation. Four bearings are installed on one shaft as shown in Fig. 15. The parameters of rolling bearing are displayed in Tab. 4. The 


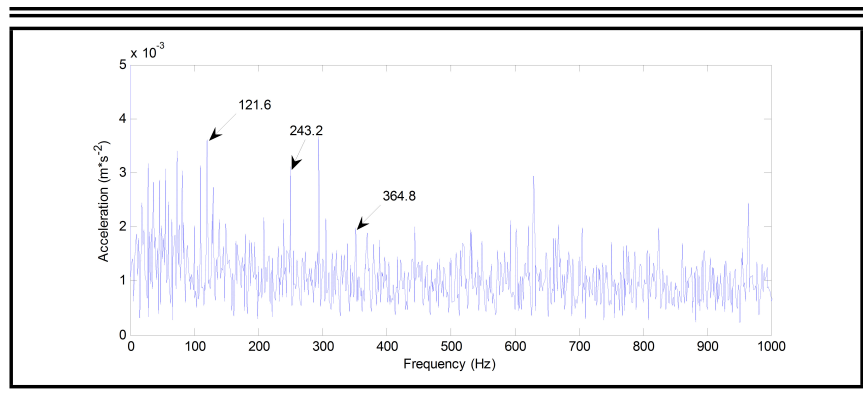

Figure 13. Filter signal TKEO frequency spectrum.

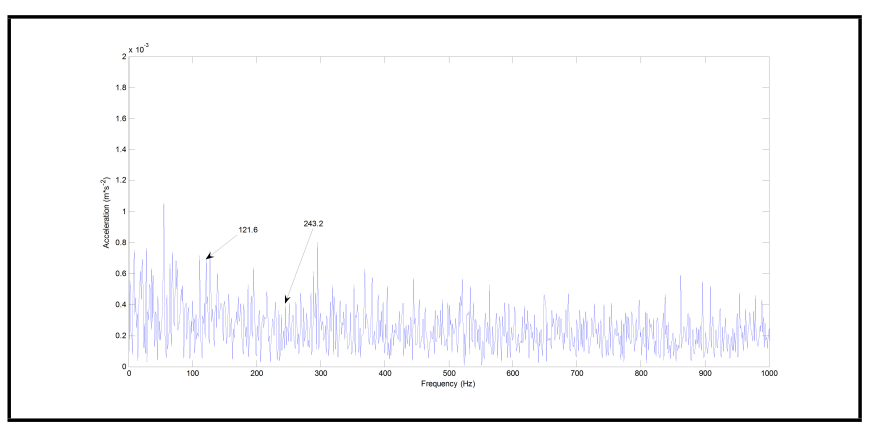

Figure 14. The spectrum of TKEO demodulation directly on the $6970 \mathrm{~min}$ data.

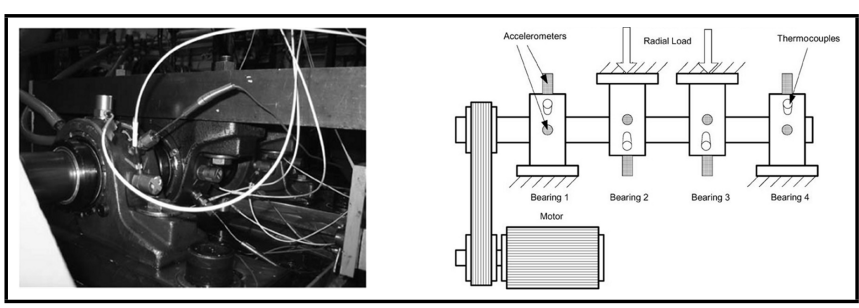

Figure 15. The bearing experiment platform and schematic diagram.

rotation speed is kept at $2000 \mathrm{rmp}$. A radial load of $26671 \mathrm{~N}$ is applied onto the shaft and bearing by a spring mechanism, and all bearings are force lubricated. The acceleration sensors are installed on bearing housing (the horizontal and vertical direction). Data collection is facilitated by NI DAQ Card 6062E.

Three data sets are included in the experimental data. The second data is chosen for outer race fault detection in the initial stage of degradation. At the end of the test-to-failure experiment, outer race failure occurred in bearing 1 . The wholelifetime of the test bearing is $164 \mathrm{~h}$. Data are collected every 10 minutes, and 20480 points are collected every time. The data sampling rate is $20 \mathrm{kHz}$. A total of 984 sets data are collected. The outer race fault frequency can be obtained as $236 \mathrm{~Hz}$.

The RMS of whole lifetime vibration data is demonstrated in Fig. 16 to search for the degradation initial stage vibration. RMS shows larger fluctuations at $7000 \mathrm{~min}$, and the fault can be detected. The RMS begins to increase at $5290 \mathrm{~min}$. Consequently, the data at this time is set as the vibration signal of the initial stage of degradation. Its time domain figure and squared envelope spectrum is shown as Fig. 17. Whether fault has occurred is not determined from the squared envelope spectrum. Fault frequency is submerged in noise, and fault can not be detected effectively.

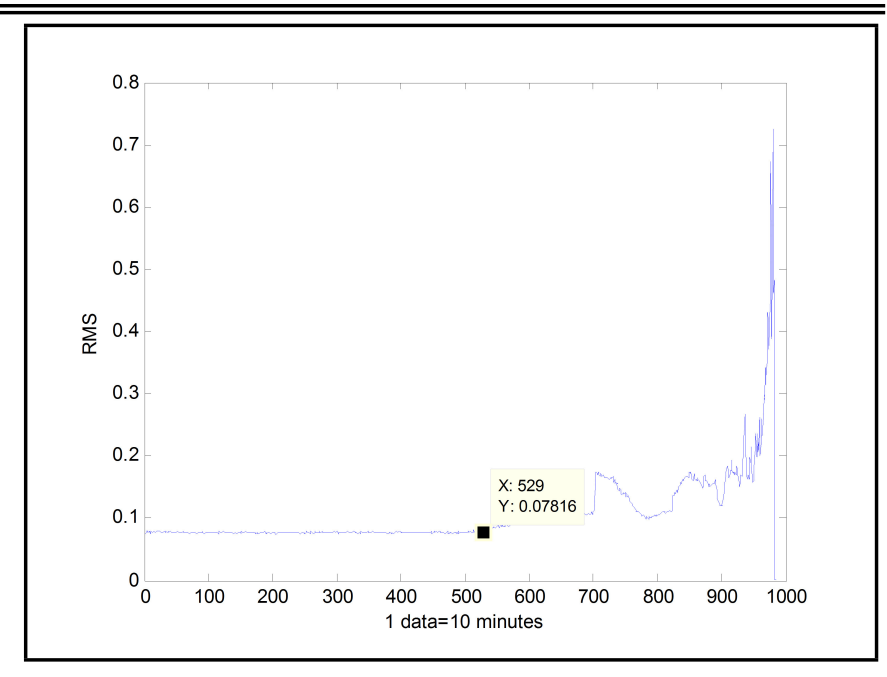

Figure 16. The RMS of whole lifetime.

Parameters of the optimizing algorithms are the same as in Section 4.2. The fitness curve of parameter optimization is shown in Fig. 18. Optimized results are demonstrated in Table 5 . The results show that the kurtosis of the denoised signal is obviously increased. The impact component has been improved accordingly.

Original vibration signal at $5290 \mathrm{~min}$ is denoised with optimized NLM. Time domain waveform after optimized NLM is manifested in Fig. 19. The denoised signal is processed with TKEO, and TKEO frequency spectrum after optimized NLM is shown in Fig. 20. The fault frequency and the doubling can be detected from the figure, and the results demonstrate the effectiveness of the proposed method in this paper.

Time domain waveform after optimized GA-NLM is also demonstrated in Fig. 21. It is arduous to find out the more pleasurable optimization method only from the time domain waveform. The denoised signal is processed with TKEO, and TKEO frequency spectrum after GA-NLM is shown in Fig. 22. The fault frequency is submerged in noise and we hardly discover fault information and it is very little if anything detects the fault. Consequently, we can determine that the denoising effect is less impressive by GA-NLM.

The corresponding kurtogram is manifested in Fig. 23 and the maximum kurtosis is $K_{1.5}$, indicated by the black rectangle in the figure. The filter signal with the maximum kurtosis is displayed in Fig. 24, and it is further processed by TKEO in Fig. 25. Although it can be detected by fault frequency, the detection effect is not as outstanding as the proposed method. Meanwhile, we hardly discover fault information in inner race fault detection in Fig. 13. This shows that the performance of FK filter is not stable, for which the six parameters of a filter can affect the detection result.

In order to explain the necessity of this method, TKEO demodulation method that is directly carried out in origin signal is taken as comparison. The spectrum of TKEO demodulation directly on the 5290min data is shown in Fig. 26, which is similar to Fig. 16(GA). The denoising effect is less impressive by GA-NLM, for which GA falls into local optimal solution. In the early stage of degradation, the fault signal is extremely weak, and TKEO is its sensitivity to noise. It is arduous to find out fault information and the fault is either little or nothing is 
Table 5. Comparison of FOA and GA optimization algorithms results.

\begin{tabular}{|c|c|c|c|c|}
\hline Algorithm & $\begin{array}{c}\text { Optimized results } \\
(p, \lambda)\end{array}$ & $\begin{array}{c}\text { Time in optimization } \\
\text { algorithms }(\mathrm{s})\end{array}$ & $\begin{array}{c}\text { Convergent iterative } \\
\text { number }\end{array}$ & $\begin{array}{c}\text { The kurtosis of } \\
\text { denoised signal }\end{array}$ \\
\hline FOA & $(42,0.115)$ & 3.4859 & 25 & 7.8365 \\
\hline GA & $(78,0.462)$ & 25.8754 & 43 & 5.2483 \\
\hline
\end{tabular}

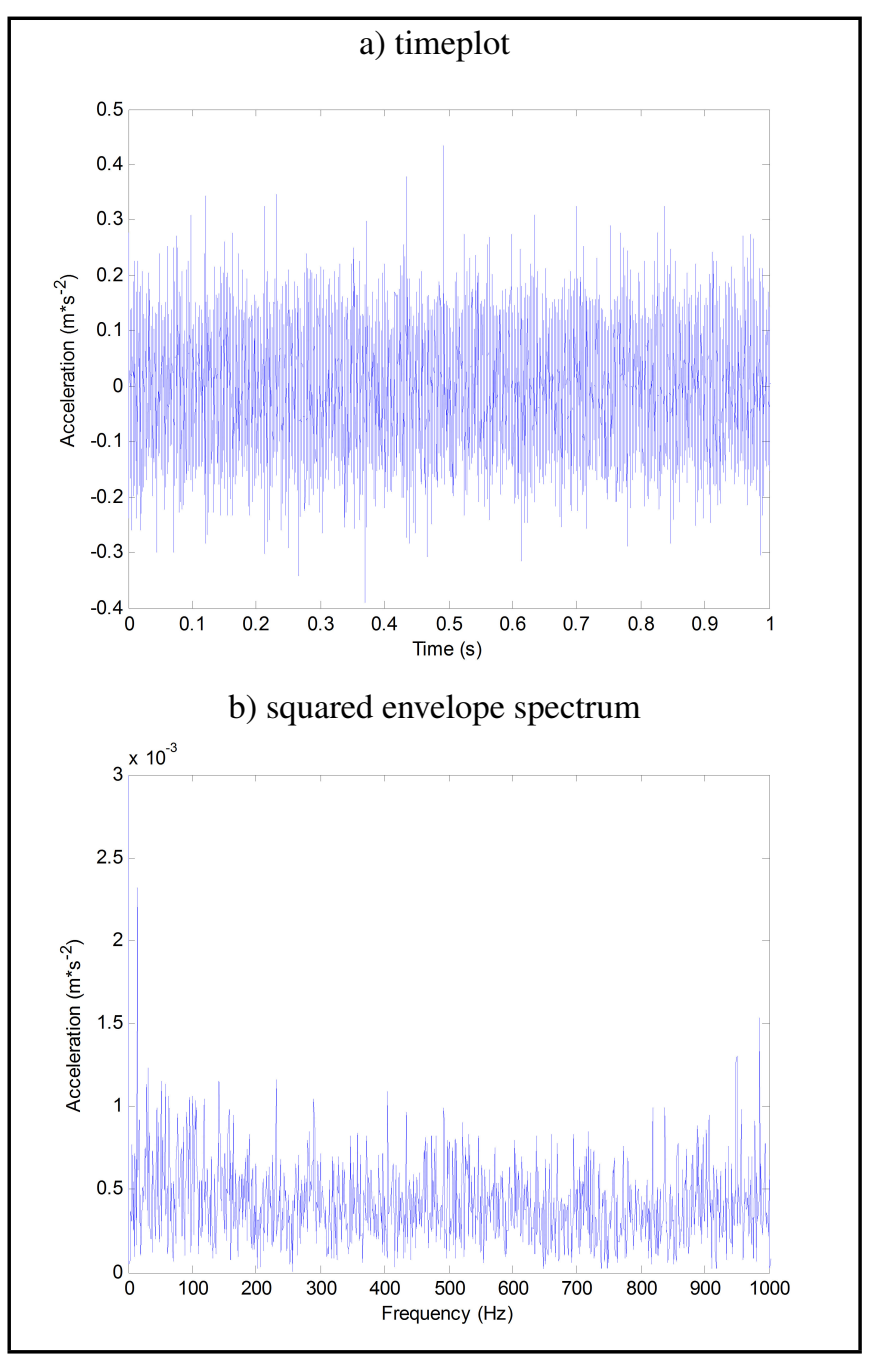

Figure 17. The time domain and squared envelope spectrum at $5290 \mathrm{~min}$.

detected as a result of fault frequency submerged in noise. Accordingly, the necessity of the proposed method is verified.

\section{CONCLUSIONS}

1. The parameter selection of NLM directly affects the denoising effect, and FOA can optimize the parameters of NLM so as to avoid the blindness of parameter selection.

2. The performance of FK filter is not stable, for the reason that the parameters of a filter can affect the detection result. The denoising effect is not as outstanding as FOANLM method.

3. The impact composition in the early degradation state of the rolling bearing is extremely weak. TKEO demodulation is difficult to detect the fault with signals whose SNR is low. The optimized NLM can achieve positive denoising effect and highlight the impact components, and TKEO can effectively detect the fault components after optimized NLM processing.

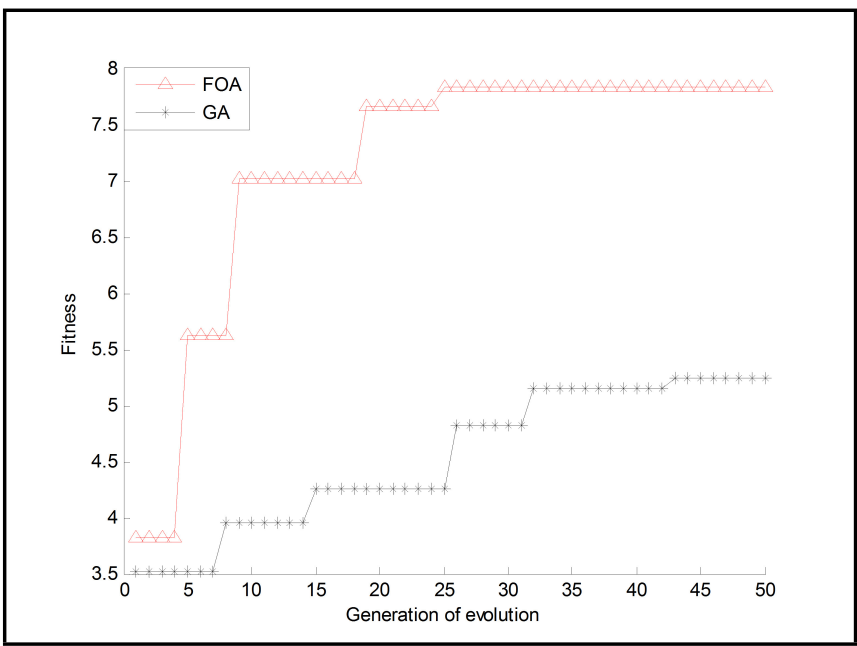

Figure 18. The fitness curve of parameter optimization.

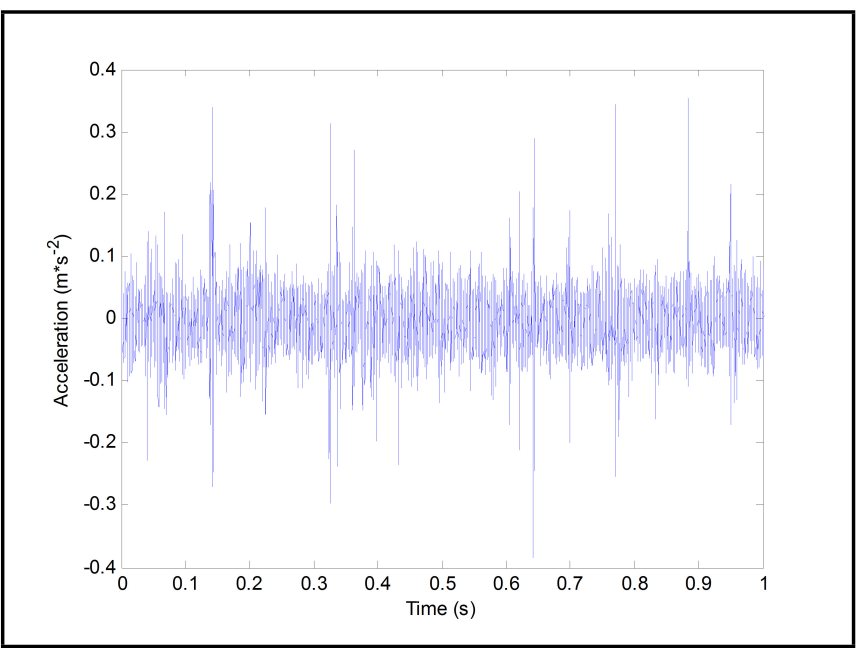

Figure 19. Timeplot after FOA-NLM.

4. The proposed method which is able to detect the fault components in the initial stage of bearing degradation is applied in two kinds of experiment data including the inner race fault one and the outer race fault one. The obtained experimental results demonstrate the effectiveness of the proposed method.

\section{ACKNOWLEDGEMENTS}

The authors are grateful to Cincinnati University Intelligent Maintenance Systems. This project is supported by National Natural Science Foundation of China (Grant No. 51541506).

\section{REFERENCES}

1 Dolenc, B., Boškoski, P., and Juričić, D. Distributed bearing fault diagnosis based on vibration analysis, Mechanical Systems and Signal Processing, 66-67, 521-532, (2016). https://dx.doi.org/10.1016/j.ymssp.2015.06.007 


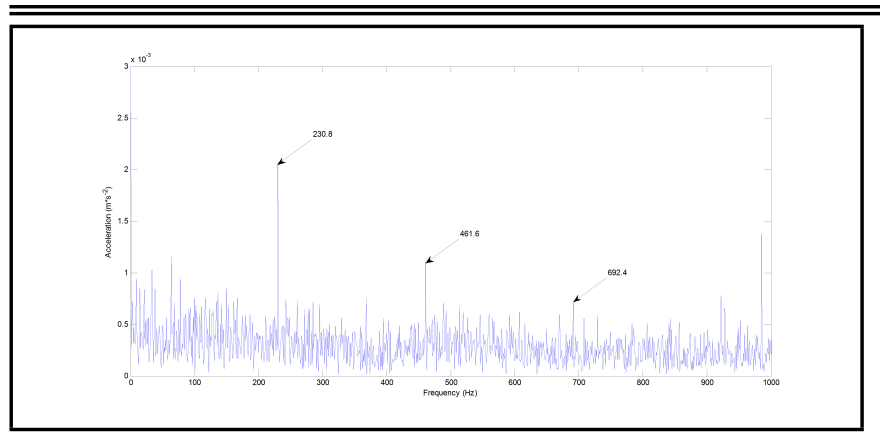

Figure 20. TKEO frequency spectrum after FOA-NLM.

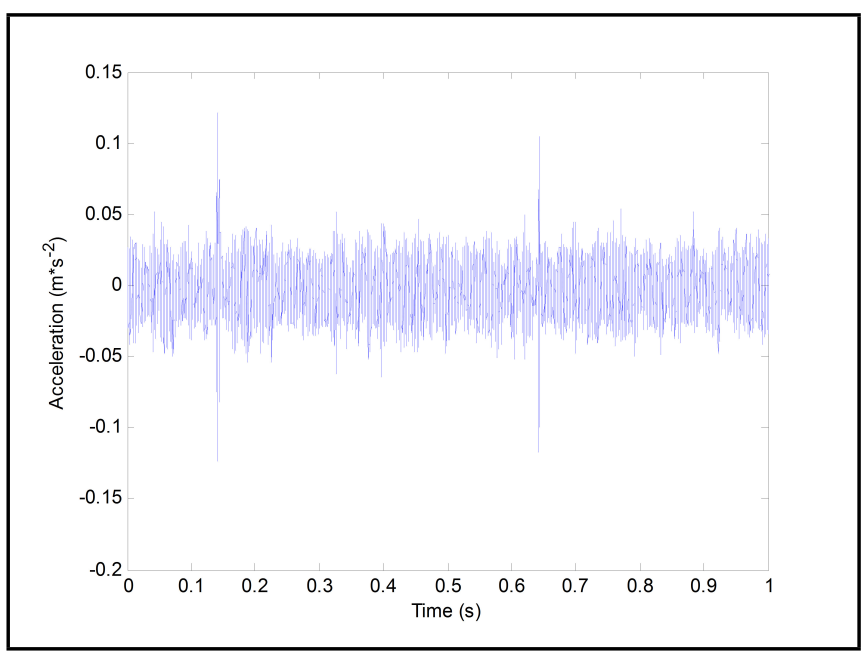

Figure 21. Timeplot after GA-NLM.

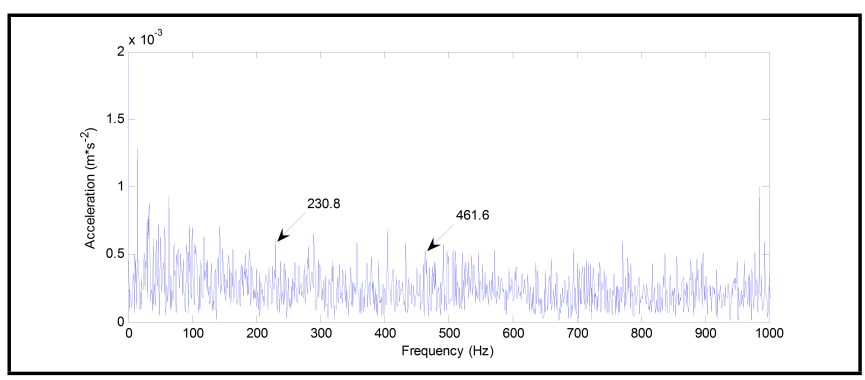

Figure 22. TKEO frequency spectrum after GA-NLM.

2 Asadzadeh, S. M. and Azadeh, A. An integrated systemic model for optimization of condition-based maintenance with human error, Reliability Engineering and System Safety, 124, 117-131, (2014). https://dx.doi.org/10.1016/j.ress.2013.11.008

3 Li, H. R., Wang, Y. K., Wang, B., et al. The application of a general mathematical morphological particle as a novel indicator for the performance degradation assessment of a bearing, Mechanical Systems and Signal Processing, 82, 490-502, (2017). https://dx.doi.org/10.1016/j.ymssp.2016.05.038

4 Park, C. S., Choi, Y. C., and Kim, Y. H. Early fault detection in automotive ball bearings using the minimum variance cepstrum, Mechanical Systems and Signal Processing, 38, 534-548, (2013). https://dx.doi.org/10.1016/j.ymssp.2013.02.017

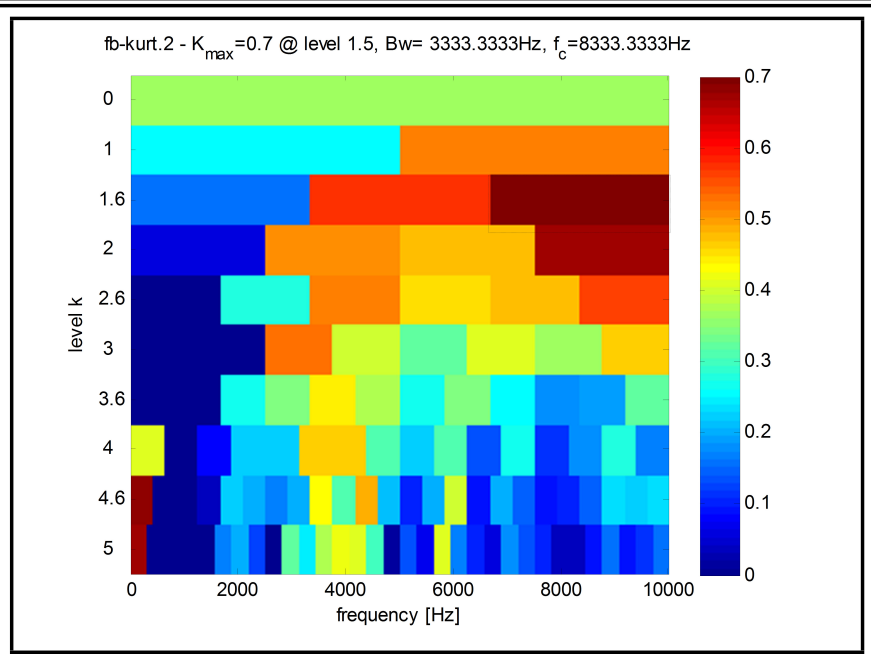

Figure 23. Kurtogram for the experimental bearing at $6970 \mathrm{~min}$.

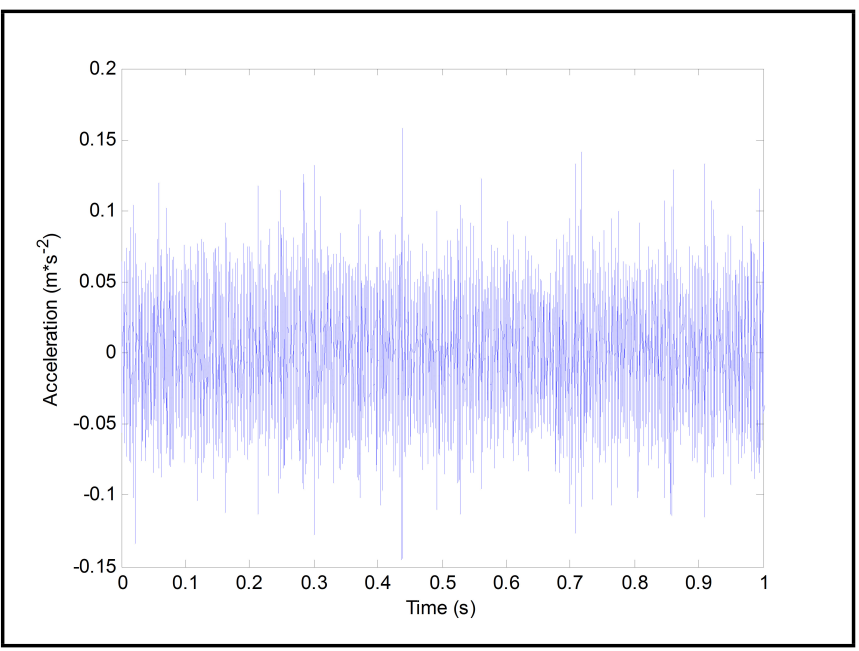

Figure 24. Filter signal timeplot.

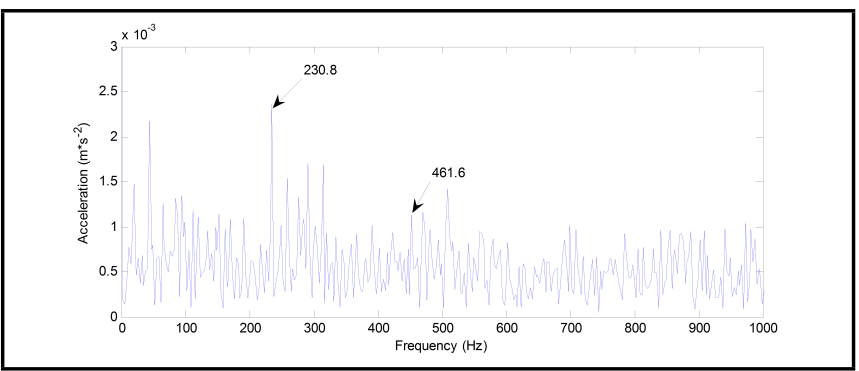

Figure 25. Filter signal TKEO frequency spectrum.

5 Antoni, J. and Randall, R. B. The spectral kurtosis: application to the vibratory surveillance and diagnostics of rotating machines, Mechanical Systems and Signal Processing, 20, 308-331, (2006). https://dx.doi.org/10.1016/j.ymssp.2004.09.002

6 Zhang, Y. X. and Randall, R. B. Rolling element bearing fault diagnosis based on the combination of genetic algorithms and fast kurtogram, Mechanical Systems and Signal Processing, 23, 1509-1517, (2009). https://dx.doi.org/10.1016/j.ymssp.2009.02.003

7 Lei, Y. G., Lin, J., He, Z. J., et al. Application of an improved kurtogram method for fault di- 


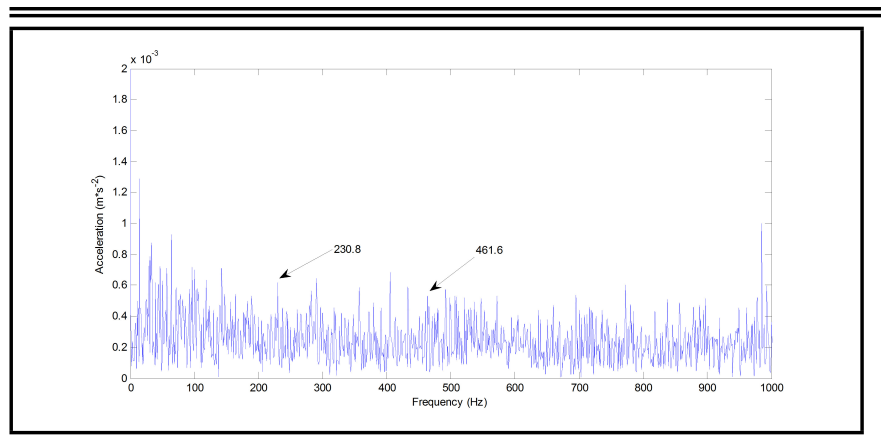

Figure 26. The spectrum of TKEO demodulation directly on the $5290 \mathrm{~min}$ data.

agnosis of rolling element bearings, Mechanical Systems and Signal Processing, 25, 1738-1749, (2011). https://dx.doi.org/10.1016/j.ymssp.2010.12.011

8 Wang, C., Zhou, J., and Liu, S. Adaptive nonlocal means filter for image deblocking. Signal Processing:Image Communication, 28, 522-530, (2016). https://dx.doi.org/10.1016/j.image.2013.01.006

9 Dolui, S., Kuurstra, A., Iván C., Salgado Patarroyo, et al. A new similarity measure for non-local means filtering of MRI images, J. Vis. Commun. Image R, 24, 1040-1054, (2013). https://dx.doi.org/10.1016/j.jvcir.2013.06.011

10 Van, M., Kang, H. J., and Shin, K. S. Rolling element bearing fault diagnosis based on non-local means de-noising and empirical mode decomposition, IET Science Measurement \& Technology, 8 (6), 571-578, (2014). https://dx.doi.org/10.1049/iet-smt.2014.0023

11 Lv, Y., Zhu, Q. L., and Yuan, R.. Fault Diagnosis of Rolling Bearing Based on Fast Nonlocal Means and Envelop Spectrum, Sensors, 15 (9), 1182-1198, (2015). https://dx.doi.org/10.3390/s150101182

12 Zhu, Q. L., Lv Y., Li N. Fault Diagnosis to Rolling Bearing by Integration of Nonlocal Means De-noising and LMD, Machine Tool \& Hydraulics, 43 (13), 172-176, (2015). https://dx.doi.org/10.3969/j.issn.1001-3881.2015.13.041

13 Zhu, K. H., Song, X. G., and Xue, D. X. A roller bearing fault diagnosis method based on hierarchical entropy and support vector machine with particle swarm optimization algorithm, Measurement, 47, 669-675, (2014). https://dx.doi.org/10.1016/j.measurement.2013.09.019

14 Pan, W. T. A new Fruit Fly Optimization Algorithm: Taking the financial distress model as an example, Knowledge-Based Systems, 26, 69-74, (2012). https://dx.doi.org/10.1016/j.knosys.2011.07.001

15 Zheng, X. L., Wang L., and Wang S. Y. A novel fruit fly optimization algorithm for the semiconductor final testing scheduling problem, Knowledge-Based Systems, 57, 95-103, (2014). https://dx.doi.org/10.1016/j.knosys.2013.12.011
16 He, Z. Z., Qi, H., Yao, Y. C., et al. Inverse estimation of the particle size distribution using the Fruit Fly Optimization Algorithm, Applied Thermal Engineering, 88, 306-314, (2015). https://dx.doi.org/10.1016/j.applthermaleng.2014.08.057

17 Rodríguez, P. H., Alonso, J. B., Ferrer, M. A., et al. Application of the Teager-Kaiser energy operator in bearing fault diagnosis, ISATransactions, 52, 278-284, (2013). https://dx.doi.org/10.1016/j.isatra.2012.12.006

18 Wang, B., Li, H. R., Chen, Q. H., et al. Rolling Bearing Performance Degradative State Recognition Based on Mathematical Morphological Fractal Dimension and Fuzzy Center Means, Acta Armamentarii, 36 (10), 1982-1990, (2015). https://dx.doi.org/10.3969/j.issn.1000-1093.2015.10.022

19 Kvedalen, E. Signal processing using the Teager Energy Operator and other nonlinear operators, Cand. Scient Thesis, (2003).

20 Buades, A., Coil, B., Morel, J. M. A review of image denoising algorithms with a new one, Multiscale Modeling \& Simulation, 4 (2), 490-530, (2005). https://dx.doi.org/10.1137/040616024

21 Van De Ville D. and Kocher M. SURE-based non-local means, IEEE Signal Processing Letters, 16 (11), 973-976, (2009). https://dx.doi.org/10.1109/lsp.2009.2027669

22 Van De Ville, D. and Kocher, M. Nonlocal means with dimensionality reduction and SURE-based parameter selection, IEEE Transactions on Image Processing, 20 (9), 2683-2690, (2011). https://dx.doi.org/10.1109/tip.2011.2121083

23 Zhang, C., Chen, J. J. Fault severity assessment for rolling element bearings based on LMD and Lempel-Ziv index, Journal of Vibration and Shock, 31 (16), 77-82, (2012). https://dx.doi.org/10.3969/j.issn.1000-3835.2012.16.016

24 Yang, Q. L., Mei, J. M., Xiao, J., et al. Weak fault feature extraction for bearings based on an order cepstrum enhanced with Teager energy operator, Journal of Vibration and Shock, 34 (6), 1-5, (2015). https://dx.doi.org/10.13465/j.cnki.jvs.2015.06.001

25 James, F. K. On a simple algorithm to calculate the energy of a signal, Proceedings of IEEE International Conference on Acoustics, Speech and Signal Processing, Albuquerque, USA, (1990). https://dx.doi.org/10.1109/icassp.1990.115702 